\title{
Prenatal Diagnosis of Megacystis Microcolon Intestinal Hypoperistalsis Syndrome:Visualisation of Microcolon
}

\author{
Mamatha Gowda ${ }^{1 *}$, Bibekanand Jindal ${ }^{2}$, Bikash Kumar Naredi ${ }^{2}$, Ketaki V Gharpure ${ }^{2}$, Nishad Plakkal ${ }^{3}$ and Laxmi \\ Godipelli $^{4}$
}

${ }^{1}$ Department of Obstetrics and Gynecology, Jawaharlal Institute of Postgraduate Medical Education and Research (JIPMER), India

${ }^{2}$ Department of Paediatric surgery, Jawaharlal Institute of Postgraduate Medical Education and Research (JIPMER), India

${ }^{3}$ Department of Neonatology, Jawaharlal Institute of Postgraduate Medical Education and Research (JIPMER), India

${ }^{4}$ Department of Obstetrics and Gynecology, Jawaharlal Institute of Postgraduate Medical Education and Research (JIPMER), India

Submission: June 05, 2018; Published: July 05, 2018

*Corresponding author: Mamatha Gowda, Assistant professor, Department of Obstetrics and Gynecology, Jawaharlal Institute of Postgraduate Medical Education and Research (JIPMER), E-38, JIPMER campusDhanvantrinagar, Gorimedu, Pondicherry, India, Tel: +91-9488127716;

Email: drmamathagowda@gmail.com

Abstract

We are reporting a case of Megacystis Microcolon Intestinal Hypoperistalsis syndrome diagnosed prenatally at 25 weeks of gestation. Fetus had bilateral hydronephrosis and megacystis in the presence of polyhydramnios suggestive of non-obstructive Megacystis. There were 2-3 parallelly arranged, elongated tubular structures with highly echogenic wall in the fetal abdomen suggestive of Microcolon. We propose this as an additional sign to be searched for in fetuses suspected to have MMIHS and may be contributory in reinforcing the diagnosis. The newborn delivered at term had severe abdominal distention with hugely distended bladder. Baby succumbed on postnatal day 15 despite palliative ileostomy. MMIHS is a rare disorder with no effective treatment available and has mostly lethal outcome. Prenatal diagnosis is difficult and can be suspected if the fetus shows hugely distended bladder, bilateral hydronephrosis with polyhydramnios suggesting a non-obstructive cause. MMIHS may have genetic etiology with autosomal recessive inheritance, hence subsequent pregnancies need to be monitored with serial ultrasounds.

Keywords: Megacystis Microcolon Intestinal Hyoperistalsis Syndrome; Prune-belly syndrome; Megacystis-microcolon; MMIHS; Hydronephrosis; Bladder outlet obstruction

\section{Introduction}

Megacystis-microcolon-intestinal hypoperistalsis syndrome (MMIHS) is a rare genetic disorder with mostly lethal outcome. Prevalence is unknown but has been reported in around 230 patients, of which $71 \%$ are females.There are few published reports and series on prenatal diagnosis of MMIHS. Fetal diagnosis is difficult because an identifiable genetic locus is absent and there are no specific ultrasound findings.Prenatal diagnosis may be possible if there is positive family history and ultrasound shows megacystis and bilateral hydronephrosis with increased liquor[1]. The fetus described by us had bilateral hydronephrosis and megacystis at 25 weeks of gestation along with polyhydramnios suggesting a non-obstructive cause. While we were speculating about the possible etiology, presence of few tubular structures with echogenic walls arranged parallel to each other suggesting microcolon in the fetal abdomen reinforced the diagnosis. The term, female baby underwent palliative ileostomy but expired postoperatively.

\section{Case Details}

A primigravida was referred in view of a cystic mass in the fetal abdomen at 25 weeks of pregnancy. Ultrasound revealed bilateral hydronephrosis and hugely distended bladder (Figure 1A). There was moderate polyhydramnios with an amniotic fluid index of $25 \mathrm{~cm}$ suggesting a non-obstructive cause for the megacystis. There were 2-3 echogenic, elongated tubular structures with highly echogenic walls arranged parallel to each other in the fetal abdomen (Figure 1B). These were suspected to be narrow segments of colon probably microcolon. Due to the combination of these findings, a provisional diagnosis ofMegacystis-Microcolon Intestinal Hypoperistalsis syndrome was made. Parents were counselled about the predicted poor 


\section{Juniper Online Journal of Case Studies}

prognosis for the fetus. As the gestation had crossed the legal limits for termination pregnancy was continued. Mother presented at 31 weeks of gestation with preterm rupture of membrane which was managed conservatively. She delivered a female baby weighing 2500g at term with an Apgar of $7 / 8$ at 1/5 minutes. Postnatally baby did not pass meconium and there was huge distension of abdomen(Figure 2A). Ultrasound abdomen of the neonate showed bilateral hydronephrosis, distended bladder and collapsed bowel loops. Neonatal echocardiography was done with normal findings. Investigation with barium meal showed microcolon. The baby was operated upon and intraoperatively the bladder was flabby and there was microcolon (Figure 2B). A palliative Ileostomy was done, however the baby expired on postnatal day 15.

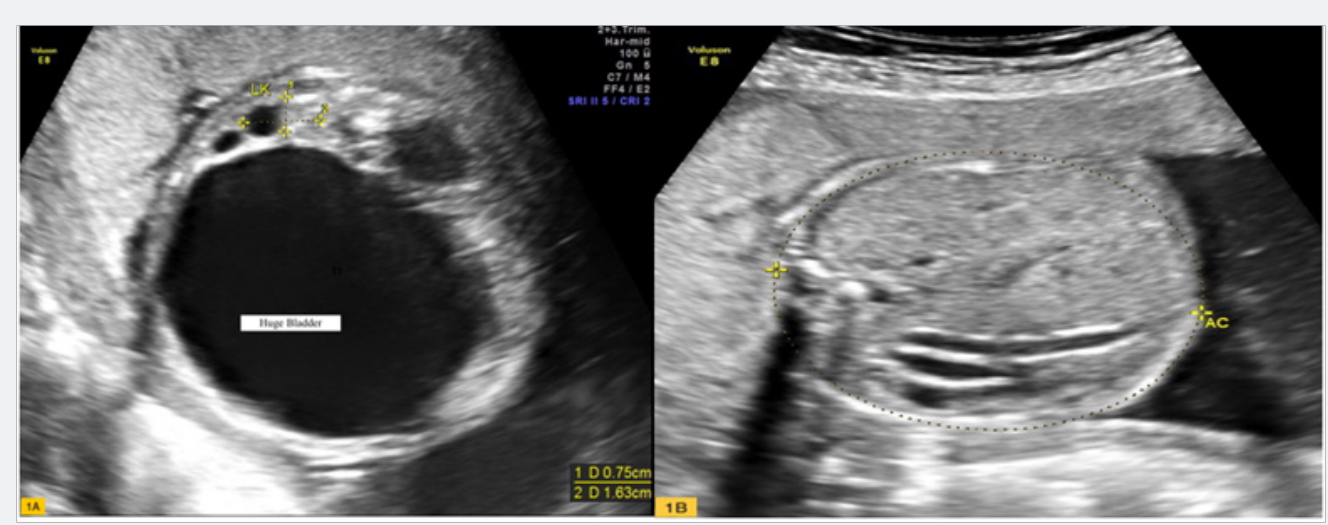

Figure 1: Prenatal ultrasound showing,

1A. Bilateral hydronephrosis and megacystis.

1B. Parallelly arranged, echogenic, elongated tubular structures with highly echogenic walls in the fetal abdomen.
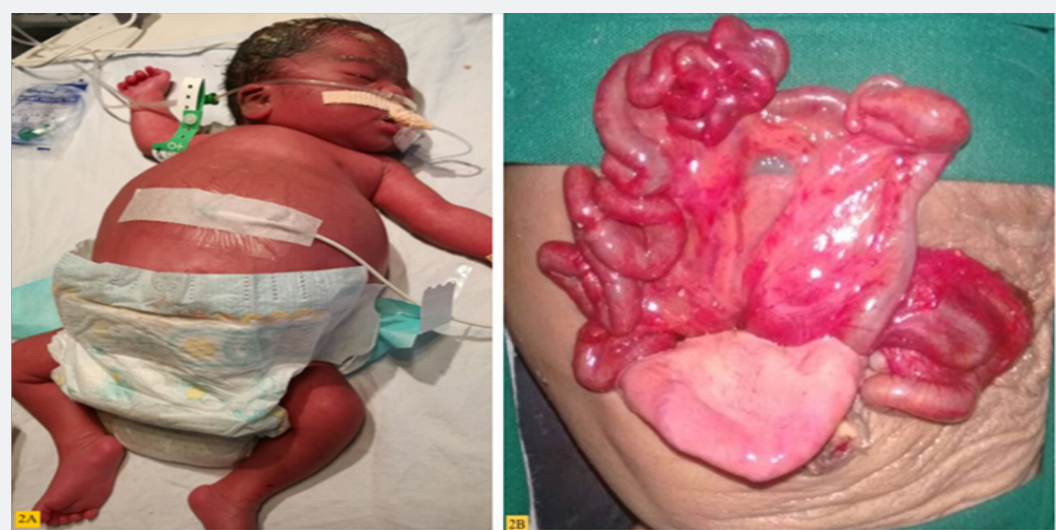

Figure 2: Postnatal findings,

2A. Prune belly appearance of neonate.

2B. Enlarged, flabby urinary bladder and microcolon.

\section{Discussion}

Megacystis-microcolon-intestinal hypoperistalsis syndrome (MMIHS) is a rare autosomal recessive disorder, showing preponderance in females and usually lethal disease during the first year of life[2].Etiology of this disorder which affects the muscle tone in the intestinal and urinary tract systems is unknown but various hypotheses are proposed including genetic, neurogenic, myogenic and hormonal causes. The presence of vacuolation and degeneration in smooth muscle of bowel and bladder wall suggest that the condition could result from an underlying visceral myopathy.The condition is often inherited in an autosomal recessive manner but may follow autosomal dominant pattern too. Heterozygousvariations in ACTG2 gene coding for smooth muscle actin was found in a cohort of 19 patients with MMIHS. Homozygousloss of function variations in MYH11 gene coding for the smooth muscle myosin heavy chain is also a probable causative factor $[3,4]$.

Prenatal diagnosis is difficult and fetuses with MMIHS frequently have megacystis and bilateral hydronephrosis with normal to increased liquor suggesting non-obstructive cause. Enlarged bladder can be observed from the second trimester and polyhydramnios from the third. Few have reported prenatal diagnosis of MMIHS based on fetal magnetic resonance imaging (MRI) in combination with analysis of enzymatic changes.A 
recent analysis of a total of 50 previously published cases of MMIHS revealed that prenatal diagnosis was achieved in $26 \%$ of these cases.In $54 \%$ of patients with a correct antenatal diagnosis there was a previously affected sibling[5].In the case described by us there was hugely enlarged bladder and bilateral hydronephrosis along with polyhydramnios. We also noticed parallel arrangement of three tubular structures with highly echogenic walls in the fetal abdomen suggesting microcolon. We suggest that this sign, if noticed during prenatal evaluation for suspected MMIHS, could be valuable to reinforce the diagnosis. PostnatallyMMIHS is diagnosed based mainly on clinical presentation and supportive radiological and surgical findings. Neonates present with non-obstructive enlargement of bladder leading to abdominal distension along and signs of intestinal obstruction such asbilious vomiting and failure to pass meconium. There can be other associated anomalies of digestive and urinary tracts such as malrotation of the gut, short bowel, renal dysplasia and cardiac anomalies.MMIHS is frequently confused with chronic idiopathic intestinal pseudo-obstruction, a milder autosomal dominant disorder in which megacystis is also present and with prune belly syndrome[2].

MMIHS has been suggested to have an autosomal recessive inheritance pattern with no specific genes identified as causative, so genetic counseling remains difficult. Prenatal evaluation of subsequent pregnancies by ultrasound in recommended in affected families as the risk of recurrence is $25 \%$ in subsequent pregnancies[1]. There was no family history in the present case, however parents were counselled about the possibility of recurrence and the need for ultrasound monitoring of future pregnancies. Till date there are no curative treatment available and palliative care with total parenteral nutrition is required in majority. Various surgical interventions such as gastrostomy, jejunostomy and vesicostomy have been tried but mostly unsuccessful. Multivisceral transplantations have been reported to improve life expectancy. Options for prenatal therapy are also limited as the renal function is well preserved and there is no oligohydramnios, hence no rationale exists for vesicoamniotic shunt.Though survival seems to have improved with specialized care, innovations in parenteral nutrition, and introduction of multivisceral transplantation, the prognosis and life expectancy of this generally fatal disease still remains poor. Death is mainly caused by sepsis, malnutrition or multiple organ failure. The neonate in our case expired within a month after birth despite starting on parenteral nutrition and performing ileostomy[6].

\section{Summarize}

MMIHS is a rare disorder with probable genetic etiology and frequently fatal within the first year of life. Prenatal diagnosis is difficult as no causative genes have been identified so far and ultrasound findings are non-specific. Fetal diagnosis by ultrasound may be possible in the presence of nonobstructive enlargement of bladder, bilateral hydronephrosis with polyhydramnios especially if there is positive family history. It may be possible to identify microcolon as narrow tubular structures in the fetal abdomen. As the condition is frequently inherited in autosomal recessive manner, subsequent pregnancies require serial ultrasound monitoring.

\section{Disclosure}

All the authors involved have not received any funding in preparing this case report. There is no funding agency involved and none of the authors have any association with any funding agencies.

\section{References}

1. Puri P (2012) Megacystic-microcolon-intestinal hypoperistalsis syndrome. Orphanet.

2. Gosemann J,Puri P (2011) Megacystis microcolon intestinal hypoperistalsis syndrome: systematic review of outcome. Pediatr Surg Int 27(10): 10411046.

3. Wangler MF, Gonzaga-Jauregui C, Gambin T, Penney S, Moss T, et al. (2014) Heterozygous De Novo and Inherited Mutations in the Smooth Muscle Actin (ACTG2) Gene Underlie Megacystis-Microcolon-Intestinal Hypoperistalsis Syndrome. PLoS Genet 10(3): e1004258.

4. Gauthier J, Ouled Amar Bencheikh B, Hamdan FF, Harrison SM, Baker LA, et al. (2015) A homozygous loss-of-function variant in MYH11 in a case with megacystis-microcolon-intestinal hypoperistalsis syndrome. Eur J Hum Genet 23(9): 1266-1268.

5. Tuzovic L, Anyane-Yeboa K, Mills A, Glassberg K, Miller R (2014) Megacystis-microcolon-intestinal hypoperistalsis syndrome: case report and review of prenatal ultrasonographic findings. Fetal Diagn Ther 36(1): 74-80.

6. Wymer KM, Anderson BB, Wilkens AA, Gundeti MS (2016) Megacystis microcolon intestinal hypoperistalsis syndrome: Case series and updated review of the literature with an emphasis on urologic management. J Pediatr Surg 51(9): 1565-1573. 
This work is licensed under Creative Commons Attribution 4.0 License DOI: 10.19080/JOJCS.2018.07.555717

Your next submission with Juniper Publishers
will reach you the below assets
- Quality Editorial service
- Swift Peer Review
- Reprints availability
- E-prints Service
- Manuscript Podcast for convenient understanding
- Global attainment for your research
- Manuscript accessibility in different formats
( Pdf, E-pub, Full Text, Audio)
- Unceasing customer service
Track the below URL for one-step submission
https://juniperpublishers.com/online-submission.php

